NIST Special Publication 1200-6

\title{
Measuring the Size of Nanoparticles in Aqueous Media Using Batch-Mode Dynamic Light Scattering
} NIST-NCL Joint Assay Protocol, PCC-1 Version 1.2

V. A. Hackley

J. D. Clogston

This publication is available free of charge from: http://dx.doi.org/10.6028/NIS T.SP.1200-6 


\title{
Measuring the Size of Nanoparticles in Aqueous Media Using Batch-Mode Dynamic Light Scattering
}

NIST-NCL Joint Assay Protocol, PCC-1

Version 1.2

\author{
V. A. Hackley \\ Materials Measurement Science Division \\ Material Measurement Laboratory \\ J. D. Clogston \\ Nanotechnology Characterization Laboratory \\ Frederick National Laboratory for Cancer Research \\ Leidos Biomedical Research, Inc. \\ Frederick, $M D$
}

November 2007 Revised May 2015

This publication is available free of charge from: http://dx.doi.org/10.6028/NIS T.SP.1200-6

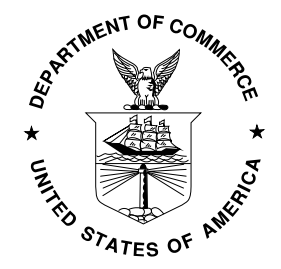

U.S. Department of Commerce Penny Pritzker, Secretary

National Institute of Standards and Technology Willie May, Acting Under Secretary of Commerce for Standards and Technology and Acting Director 
Certain commercial entities, equipment or materials may be identified in this document in order to describe an experimental procedure or concept adequately. Such identification is not intended to imply recommendation or endorsement by the National Institute of Standards and Technology, nor is it intended to imply that the entities, materials or equipment are necessarily the best available for the purpose.

National Institute of Standards and Technology Special Publication 1200-6 Natl. Inst. Stand. Technol. Spec. Publ. 1200-6, 17 pages (May 2015) CODEN: NSPUE

This publication is available free of charge from: http://dx.doi.org/10.6028/NIST.SP.1200-6 


\section{Forward}

This special publication is one in a series of protocols resulting from a collaborative research agreement between the National Institute of Standards and Technology (NIST) and the Nanotechnology Characterization Laboratory (NCL) at the National Cancer Institute's Frederick National Laboratory for Cancer Research. The original version of this protocol (PCC-1, Ver. 1.0) was first posted on the NCL web site (http://ncl.cancer.gov/working assay-cascade.asp), and it, and any other previous versions, are superseded by this updated special publication version. SP 1200-6 is technically and procedurally consistent with PCC-1 (Ver. 1.1) currently posted on the NCL assay cascade, with minor informational updates. Additionally, a video version of this protocol is available for viewing at NCL (http://ncl.cancer.gov/video.asp).

Updates to this protocol may be released in the future. Visit http://nist.gov/mml/npmeasurement-protocols.cfm to check for revisions of this protocol or new protocols in the series.

NIST and NCL are interested in soliciting feedback on this method. We value user comments and suggestions to improve or further validate this protocol. Please send your name, email address, and comments/suggestions to nanoprotocols@ nist.gov. We also encourage users to report citations to published work in which this protocol has been applied. 


\section{Introduction}

This assay protocol outlines procedures for sample preparation and the determination of mean nanoparticle size (hydrodynamic diameter) using batch-mode dynamic light scattering (DLS synonyms include photon correlation spectroscopy, PCS, and quasi-elastic light scattering, QELS) in dilute aqueous suspensions. The hydrodynamic diameter is the diameter of an equivalent hard sphere that diffuses at the same rate as the analyte. Although particle size is the primary determinant of the measured diffusion coefficient, other parameters can impact these measurements and influence the measured size. Therefore, guidelines for making successful size measurements in the nanosize range are provided, as well as a discussion of relevant standards and data analysis. This protocol can be applied to any suitable DLS instrument with batch measurement capability. This is intended to be an evolving document; addenda that address material-class-specific sample preparation and analysis issues will be appended in the future.

\section{Sample Preparation and Measurement Procedure}

2.1 Always wear appropriate personal protective gear (e.g., gloves, lab coat, goggles, respirator, etc.) and take appropriate precautions when handling your nanomaterial.

2.2 Measurement cuvettes should be cleaned with filtered demineralized water and stored dry. Periodic use of commercial cleaning agents formulated specifically for optical cells and components is recommended to remove difficult residues, but care must be taken to remove all traces of the cleaning detergent as this may impact the nanomaterial properties. Keep cleaned cuvette sealed/capped until needed. If available, store cuvette under HEPA filtered air (e.g., in a clean bench).

2.3 Suspending medium (a.k.a, solvent, dispersant, solution) should be filtered prior to sample preparation using a $0.1 \mu \mathrm{m}$ or smaller pore size membrane, and should be tested for scattering contributions to the measured signal in the absence of the analyte. As a general rule, one should filter the medium to at least the nominal size of the analyte to be measured. This may not be practical for particles smaller than $20 \mathrm{~nm}$, and so the suspending medium should always be measured separately to ensure the absence of, or to account for, interfering particles or nanoscale surfactant structures present at significant concentrations.

2.4 A typical starting sample concentration is $1 \mathrm{mg} / \mathrm{mL}$. This concentration should be adjusted to accommodate the scattering properties of your sample and/or the optical requirements of your specific instrument (i.e., according to instrument manufacturer specifications for acceptable count rate range).

2.5 Use cuvette with quartz or optical-quality glass windows. Choose the appropriate cuvette capacity depending on your available sample volume and instrument requirements. Pre-rinse cuvette with filtered solvent at least 3 times.

Note: Plastic disposable cuvettes are convenient for high throughput analysis, and can be a significant time-saver, as they can be used once and discarded. However, the optical quality of disposable cuvettes is generally poor compared to quartz or glass, and 
therefore their efficacy for a particular type and concentration of nanoparticles should be validated prior to use. Generally, disposable cuvettes are less prone to artifacts as the particle size, particle concentration and/or particle refractive index increases.

Comparison of results obtained using disposable and quartz or glass cuvettes is recommended. Plastic cuvettes also scratch easily, and care should be taken to avoid abrading the surface (e.g., with a laboratory chemical wipe).

2.6 Samples should be filtered with a $0.2 \mu \mathrm{m}$ or smaller pore size membrane, preferably in conjunction with loading into the cuvette. The choice of pore size depends on the maximum dimension of the test particles and their tendency to adhere to the filter membrane. This is discussed along with non-filtration alternatives, in section 3.4. Whichever procedure is used, it should be validated for the test material prior to measurement to ensure that the analyte is not being removed or otherwise modified by the process.

\subsection{Loading Sample into the Cuvette}

2.7.1 Pre-rinse filter with solvent (at least $1 \mathrm{~mL}$, depending on filter size and dead volume of filter holder or cartridge).

2.7.2 After loading syringe with sample and inserting syringe filter, allow the first 4 drops to go to waste. If possible, use the next 4 drops to pre-rinse the cuvette, and discard. The remainder can be used for the sample measurement.

2.7.3 Load sample into cuvette using minimum amount necessary to ensure liquid level is at least $2 \mathrm{~mm}$ above the entrance height of the laser beam for your particular instrument configuration. Typically, the beam center height is either $8.5 \mathrm{~mm}$ or $15 \mathrm{~mm}$ from the cuvette bottom. Refer to the instrument manual or contact manufacturer to confirm beam height at the cuvette. For microcuvettes with a sample well insert, fill the well with sample, but do not fill beyond the well lip. Overfilling of a cuvette can lead to thermal gradients that will adversely impact measurement accuracy.

2.7.4 Take care not to touch the cuvette windows with your bare hands while loading. Wipe outside of quartz or glass cuvette with lens paper if needed.

2.7.5 Cap the cuvette to prevent dust contamination and solvent evaporation.

2.7.6 Inspect the cuvette to ensure that air bubbles are not clinging to the optical window area. If necessary, gently tap cuvette on a non-metallic padded surface to release bubbles before placing cuvette in the sample holder. Never shake cuvette, as this may introduce air bubbles or entrap air in the sample well of microcuvettes. Make sure to place the cuvette correctly in the sample holder (i.e., quartz windows should be facing the incident beam and detector).

2.7.6.1 Degassing the medium used to prepare samples (e.g., by applying a vacuum) can help prevent the formation of air bubbles during filtration and analysis. 
2.8 For a nominal sample volume of $1 \mathrm{~mL}$ or smaller, an equilibration time of 4 minutes at each temperature is recommended prior to starting measurements. If operating close to room temperature $\left(\Delta \mathrm{T} \pm 1^{\circ} \mathrm{C}\right)$, this time may be reduced by one half. Larger sample volumes may require longer equilibration times. The temperature should be controlled and measured with an accuracy and precision of $0.3{ }^{\circ} \mathrm{C}$ or better.

2.9 Perform 3 to 10 independent measurements per sample per temperature setting to establish measurement repeatability. Measurement duration should be set according to instrument manufacturer recommendations, and will differ depending on particle size and scattering characteristics, as well as the optical characteristics of the instrument itself (e.g., detector sensitivity, scattering angle, etc.). A minimum duration (measurement time) of $60 \mathrm{~s}$ is recommended for nanosize particles.

\section{Precautions and Guidelines}

\subsection{Strongly Absorbing (Colored) Samples}

Recording the visible transmission spectrum of your sample is recommended if it is colored. Check to see if your sample absorbs strongly at the DLS instrument's laser wavelength. If this is the case, use of different size-measuring instrument or laser may be necessary, or sample concentration may be increased.

\subsection{Sample Concentration}

The typical starting sample concentration is $1 \mathrm{mg} / \mathrm{mL}$, but this value should be modified accordingly to account for the scattering properties of your sample (e.g., intensity indicated by the average count rate at the detector). Scattering intensity will depend on particle size, refractive index and concentration of your sample; only the latter can be modified for analysis. Too low a concentration may seriously degrade the signal-to-noise ratio and subject the analysis to the effects of extraneous particles that might be present. This will result in noisy and inconsistent results. On the other hand, too high a concentration can lead to multiple scattering effects and particle interactions, both of which can produce changes in the measured (apparent) hydrodynamic size.

It is therefore recommended to execute a validation procedure for measurement of a given type of sample in a given instrument, in which multiple concentrations are analyzed, in order to bracket the target concentration (over at least a decade in concentration range) to ensure that the measured size is not concentration dependent or to establish the applicable concentration range. This procedure is also recommended in ISO13321:1996. In the case of an observed concentration effect, extrapolation of the apparent measured size to zero concentration may be appropriate in order to obtain a concentration independent (unbiased) size. Instrumental approaches to diminish the effects of multiple scattering include use of backscatter optics with an adjustable measurement depth or implementation of a cross-correlation technique.

The user is reminded that by diluting a sample (e.g., to obtain reduced particle concentrations) the effective particle size may change due to changes in the chemistry of the medium or changes 
in the electrical double layer of charged particles. It is therefore generally recommended for dilution purposes to use conductivity matching, a compositionally equivalent medium, or to use the sample supernatant extracted after centrifugation of the analyte.

\subsection{Effect of Salt Concentration}

The hydrodynamic size derived from DLS can depend on the salt concentration of the suspending medium. This effect arises due to the electrical double-layer surrounding charged particles in an aqueous medium. At low salt concentrations (e.g., in deionized water), the additional drag induced by the extension of the double-layer into adjacent bulk solution causes a decrease in the diffusion coefficient and an apparent increase in size. This effect tends to increase with decreasing actual particle size. Adding a small amount of inert "supporting" monovalent electrolyte (e.g., $10 \mathrm{mmol} \cdot \mathrm{L}^{-1} \mathrm{NaCl}$ ) to screen the double-layer, will eliminate this issue for most systems.

A similar effect is the hindered diffusion that occurs at high particle concentrations due to interaction between neighboring particles. This effect is enhanced by low salt concentrations and can lead to an apparent increase in particle size.

On the other hand, too high a salt concentration (e.g., $>50 \mathrm{mmol} \cdot \mathrm{L}^{-1} \mathrm{NaCl}$ ) may destabilize charged particles leading to salt-induced aggregation and an increase in both particle size and polydispersity. It is generally good practice to perform DLS measurements with some inert monovalent electrolyte present, whereas pure deionized or distilled water should generally be avoided as a dispersion medium or used for qualitative comparisons only.

The conditions under which a supporting electrolyte is used should be validated for your specific test material.

\subsection{Dust Contamination and Sample Cleanliness}

The intensity of light scattered by nanosize particles is proportional to the square of the molecular mass or $\mathrm{d}^{6}$, where $\mathrm{d}$ is the particle diameter; thus larger particles will scatter much more strongly than smaller particles. Additionally, water, as a polar solvent, acts as an excellent medium for "dust" particles (a collective term used here to refer to any foreign particulate matter originating from normal environmental contact). Although state-of-the-art DLS instruments are equipped with some form of "dust rejection" algorithm, experience shows that the best practice to achieve good reproducibility is to eliminate dust prior to analysis. This becomes especially important for samples that scatter weakly due to extremely small size and/or low refractive index contrast with the medium. Cuvettes, sample vials, solvent bottles, etc., should remain closed as much as possible to minimize contamination. Dispersion media should be filtered to a $0.1 \mu \mathrm{m}$ pore size. The solvent storage container should, if possible, be outfitted with an air intake filter with a $1 \mu \mathrm{m}$ or smaller pore size. It is recommended that the dispersion medium should be periodically checked for background scattering; ensure that it is within the instrument guidelines and record its average count rate for future comparison.

Additionally, your prepared sample should be filtered to remove large interfering particulate matter: (a) when contamination may be associated with the source material rather than the 
solvent, (b) if the sample to be analyzed was not prepared under clean conditions as specified above or (c) the sample is of uncertain origin. This should be done by selecting an appropriate filter membrane and pore size such that the principal analyte is not removed from the solution or otherwise modified during filtration. For many aqueous applications, a $0.2 \mu \mathrm{m}$ detergent-free polycarbonate or polyethersulfone filter will suffice, where the latter is a good choice for low protein binding requirements. For extremely small hydrodynamic sizes $(<5 \mathrm{~nm}$ diameter $)$, where the scattered intensity is very low, a $0.02 \mu \mathrm{m}$ pore size may be necessary to remove all interfering extraneous particles. For test particles that bind strongly to filters, use a larger pore size or try a different membrane material to reduce interaction with the filter surface.

One should always validate the filtration procedure for a material or material class prior to proceeding with the analysis. Sample filtration may only be necessary if preliminary DLS results indicate the presence of contaminating large particles.

Centrifugation is an alternative to filtration for removing large size contaminants. The parameters for correct use (speed and duration) depend on the specific rotor and tube used, and thus can be more difficult to stipulate in advance. Centrifugation is recommended for extremely small low-density particles (e.g., bovine serum albumin or other folded proteins), where adsorption by the filter may be an issue, or low generation-number dendrimers. Dust particles can be removed by spinning at moderate speeds (e.g., $4000 \mathrm{rpm}$ or $419 \mathrm{rad} / \mathrm{s}$ ) for 30 minutes in a micro-centrifuge tube without affecting the analyte. Centrifugation may also be preferred in situations where emulsions or other "soft" particles exist, which can deform or disintegrate to pass through a membrane and then reform afterwards.

Another option to minimize dust contamination is to perform sample preparation and transfer operations within a HEPA-filtered environment, and to seal the sample cuvette against further contamination prior to removing it from the clean area for analysis.

\subsection{Handling of Measurement Cuvettes}

Minimize the time uncapped cuvettes are exposed to the ambient environment so as to reduce the likelihood of dust contamination. Handle cuvettes in a manner that avoids unnecessary contact with any cuvette surface that is in the pathway of the incident or scattered laser beam. It is recommended to wear non-powder-containing latex or nitrile gloves at all times when handling the cuvettes. Cuvettes should be inspected periodically for surface scratches or coatings that might interfere with optical measurements. Use only good quality lens paper to wipe/dry external surfaces and non-abrasive particle-free clean room swabs to wipe internal surfaces.

It is not recommended to use ultrasonics to clean quartz or glass cuvettes used for optical measurements. This can degrade the surface finish or cause the cuvette to crack at joints. Cuvette suppliers offer specially formulated cleaning solutions that can be used to remove difficult residues, such as proteins.

If possible, dry the cleaned cuvettes under HEPA-filtered conditions. Extraneous dust can be removed from dry cuvettes by applying a short-duration blast of filtered non-reactive gas, e.g., from a pressurized air canister of the type typically used in clean room applications. 
As a general rule, always remove your sample from the cuvette as soon as possible following the measurements, and immediately rinse the cuvette with your filtered medium or with filtered demineralized water. Never allow a sample to dry in the cuvette. Disposable cuvettes should be rinsed and disposed of according to your laboratory's safety policy for materials that have had contact with nanoparticles.

\subsection{Instrument Warm Up}

The instrument should be powered up at least 30 minutes in advance of measurements to allow the laser to stabilize (note: stability may be less of an issue for diode lasers compared with gas lasers such as the commonly used $\mathrm{He}-\mathrm{Ne}$, but as a matter of practice this procedure ensures stability of the instrument). Additional time may be needed to bring the sample holder up to the desired temperature if the measurement temperature is not set at the time of power-up.

\subsection{Measurements}

A minimum of 3 measurements 60 s or longer in duration and up to 10 measurements per sample is recommended (ISO 13321:1996 recommends 6 replicate measurements, but this has been revised down in ISO 22412:2008). Check the raw correlation data to ensure that the amplitude (y-intercept) is stable and the correlograms are smooth (i.e., decay exponentially to a flat baseline). Noisy correlograms and/or fluctuating amplitudes for a given sample can be attributed to the presence of dust/foreign particles in the sample, concentration variations from sample precipitation or aggregation, thermal convection in poorly equilibrated solutions, solvent evaporation (if measuring at temperatures greater than ambient temperature), or dirty cuvettes.

The repeatability for a set of $\mathrm{N}$ replicate measurements, defined as $100 \cdot s /\left\langle x_{P C S}\right\rangle$ (where $\mathrm{s}$ is the estimated standard deviation at $N-1$ degrees of freedom, $\left\langle x_{P C S}\right\rangle$ is the mean z-average size, and brackets indicate an ensemble average), should be better than $5 \%$ for samples other than the reference material used to qualify the instrument performance.

If sediment is visible at the bottom of the cuvette following measurement, then the data should be discarded; sediment indicates that the sample either contains a significant portion of large (micrometer) size particles or the target particles are unstable during the time-frame of the experiment. DLS is applicable only to particles that undergo Brownian diffusional motion and remain fully suspended throughout the measurement; samples containing larger size particles should be analyzed using other techniques, such as laser diffraction, electrical sensing zone method or gravitational sedimentation.

\subsection{Waste Disposal}

Follow your facility's recommended disposal procedure for your specific nanomaterial. Most instruments will accommodate microcuvettes, which can help reduce the overall volume of waste material. 


\section{Performance Verification and Data Analysis}

\subsection{Standards and Performance Verification}

DLS is a first-principles measurement technique, and as such does not require calibration in the usual sense of the word. However, it is recommended that users periodically run standards to provide qualification (i.e., verification) of correct instrument operation within manufacturer specifications and to validate your measurement procedure.

For this purpose, latex size standards down to nominally $20 \mathrm{~nm}$ (e.g., NIST-traceable polystyrene spheres) are available from a number of commercial suppliers. For standards below $20 \mathrm{~nm}$, proteins such as cytochrome $\mathrm{c}$ and BSA are recommended. These materials are also commercially available, are relatively inexpensive, and their hydrodynamic size has been thoroughly characterized and reported in the scientific literature. Colloidal gold is commercially available in a wide range of sizes down to nominally $2 \mathrm{~nm}$. Certified reference materials are also available from NIST (https://srmors.nist.gov/), including SRMs 1963a and 1964 (100 nm and $60 \mathrm{~nm}$ polystyrene lattices) and RMs 8011,8012 and 8013 (10 nm to $60 \mathrm{~nm}$ colloidal gold), and from the European Commission's Institute for Reference Materials and Measurements (IRMM, http://irmm.jrc.ec.europa.eu), including ERM-FD100 and ERM-FD304 (colloidal silica, $20 \mathrm{~nm}$ and $40 \mathrm{~nm}$, respectively).

ISO 22412:2008 recommends that a polystyrene latex with narrow size distribution and average diameter as measured by DLS of about $100 \mathrm{~nm}$ be used for qualification purposes on a periodic basis. The measured average diameter (z-average size) of the latex sample should be within $2 \%$ of the stated size and the repeatability should be better than $2 \%$; additionally, the polydispersity index should be less than 0.1 . Deviations beyond the above stated limits indicate that a problem may exist with the instrument performance, the measurement cell, or the water used to dilute the standard prior to measurement; in this case, address possible sources of error and contact the manufacturer if cell or water issues prove inconsequential. All water-dispersed standards are subject to instabilities over time and shelf-life limitations. Check that your standard of choice has not exceeded the stated expiration date.

Particle size results obtained from DLS measurements may not coincide with those obtained from other techniques (e.g., electron microscopy). This is due in part to differences in the weighted averages determined in each case (e.g., number versus intensity), as well as differences in the physical property that is actually measured (e.g., hydrodynamic diffusion versus projected area). DLS is sensitive to the presence of small quantities of large particles or clusters of smaller particles, whereas electron microscopy, for instance, typically reflects the size of primary particles and may not include a statistically relevant sampling of larger clusters or particles.

\subsection{Data Analys is and Measurement Parameters}

A detailed description of the data analysis will not be given here. There are many methods available to analyze the raw autocorrelation data in order to extract a size distribution (see for an overview Johnson and Brown, 1992), and these methods will process the data differently using different inherent assumptions. It is left for the reader to check the instrument's user manual to 
identify the data analysis algorithm or to seek third party software solutions. Widely used methods include CONTIN and Non-Negative Least Squares (NNLS). Basic guides to DLS and data analysis can be found in the References section.

A few issues regarding data analysis are worth mentioning. Whichever model the instrument software uses to fit measured correlation data, the Stokes-Einstein equation will ultimately be employed to calculate the hydrodynamic diameter, $\mathrm{d}_{\mathrm{H}}$ :

$$
d_{H}=k T / 3 \pi \eta D
$$

where $\mathrm{k}$ is the Boltzmann constant, $\mathrm{T}$ is the temperature, $\eta$ is the absolute zero-shear viscosity of the medium and $\mathrm{D}$ is the diffusion coefficient. Thus it is important to input the correct temperature and viscosity values. Temperature is usually handled automatically by the instrument software, and good temperature control of the sample is critical for accurate measurement. On the other hand, viscosity is typically a user input value. One should therefore input correct values of viscosity for the particular solvent used at the measurement temperature. Generally, for maximum accuracy, viscosity values should be accurate to within about $1 \%$. For solutions containing significant amounts of salt (e.g., buffers, isotonic solutions), viscosity will be slightly dependent on salt concentration. For instance, use of bulk water viscosity for a sample dispersed in phosphate buffered saline (PBS) results in an error of about $2 \%$ in the viscosity at room temperature.

Similarly, it is important to input the correct refractive index (RI) for the suspending medium, as this value is used in the primary calculations yielding the diffusion coefficient. RI values should be accurate to within about $0.5 \%$, or to two decimal places for water. There is a slight dependence of RI on salt concentration (and other non-light-absorbing soluble additives) in dilute aqueous solutions, but the difference between PBS, for instance, and water, is less than $0.2 \%$. Similarly, the temperature dependence of the RI is very weak over the normal measurement temperature range for water, and varies only about $0.2 \%$ from $20^{\circ} \mathrm{C}$ to $37^{\circ} \mathrm{C}$. RI is also wavelength dependent, but for water this dependence is sufficiently weak over the normal range of wavelengths used in DLS instruments (typically in the $488 \mathrm{~nm}$ to $750 \mathrm{~nm}$ range), that values reported for the sodium $\mathrm{D}$ line $(589.3 \mathrm{~nm})$ are usually acceptable (with an error of less than $0.3 \%$ over the entire range). The RI for pure water at $20^{\circ} \mathrm{C}$ is 1.332 (calculated based on IAPWS 1997), and this value can be used for most dilute salt solutions over the temperature range from $20^{\circ} \mathrm{C}$ to $37^{\circ} \mathrm{C}$. The refractive index for the particle phase is only required for transformation of size distribution results from an intensity-weighted basis to a volume- or number-weighted basis; it does not impact the calculation of the diffusion coefficient or the $\mathrm{z}$ average size.

For extremely small particles, where all physical dimensions are smaller than about $60 \mathrm{~nm}$ (based on use of He-Ne or similar wavelength lasers), the angular dependence of scattering is negligible and the RI value input for the particles will not greatly affect the transformation to a volume-weighted distribution using the Mie model or Rayleigh-Gans-Debye (RGD) approximation (i.e., the exact value of the particle RI is not critical). On the other hand, this does not indicate that the model employed is appropriate for the particles being analyzed. 
For the reader's convenience, the table below provides recommended values for the absolute viscosity and refractive index as a function of temperature and solvent composition for several common aqueous suspending media. Viscosity is given in SI units of $\mathrm{mPa} \cdot \mathrm{s}$, which are equivalent to the c.g.s. units of centi-Poise (cP). The base values for the viscosity of pure water are derived from the NIST Chemistry WebBook (2005). The contributions of dissolved salt to the viscosity of pure water are assumed to be additive, and are based on values interpolated from data available in the CRC Handbook of Chemistry and Physics (2006) and fit using a $3^{\text {rd }}$ order polynomial. Therefore, to calculate the viscosity of a salt solution presented in the table below, at a different temperature between $0{ }^{\circ} \mathrm{C}$ and $100{ }^{\circ} \mathrm{C}$, simply subtract the value of pure water at $20{ }^{\circ} \mathrm{C}$ from the corresponding value for the salt solution. This will provide the incremental increase in viscosity for a particular salt composition. The incremental value can then be added to the viscosity for pure water at temperatures not reported below, but available from sources such as the CRC Handbook or the NIST WebBook.

\begin{tabular}{|l|c|c|c|c|}
\hline \multirow{2}{*}{ Aqueous Medium } & \multicolumn{3}{|l|}{ Absolute Viscosity $(\mathbf{m P a} \cdot \mathbf{s})$} & \multirow{2}{*}{ Refractive Index } \\
\cline { 2 - 4 } & $\mathbf{2 0}^{\mathbf{}} \mathbf{C}$ & $\mathbf{2 5}^{\mathbf{0}} \mathbf{C}$ & $\mathbf{3 7}^{\mathbf{0}} \mathbf{C}$ & \\
\hline pure water & 1.002 & 0.890 & 0.692 & 1.332 \\
$10 \mathrm{mmol} \cdot \mathrm{L}^{-1} \mathrm{NaCl}$ & 1.003 & 0.891 & 0.693 & 1.332 \\
isotonic saline $\left(154 \mathrm{mmol} \cdot \mathrm{L}^{-1} \mathrm{NaCl}\right)$ & 1.020 & 0.908 & 0.710 & 1.334 \\
phosphate buffered saline $(\mathrm{PBS})$ & 1.023 & 0.911 & 0.713 & 1.334 \\
\hline
\end{tabular}

A final note regarding data analys is: Presently, the only broadly recognized standard for the analysis of measured autocorrelation data is the cumulants method as stipulated in ISO 13321:1996 Annex A. Cumulants analysis yields a mean intensity-weighted size (commonly called the z-average diameter but identified in the standard as the average PCS diameter, XPCS, or the harmonic z-average) and a measure of the broadness of the distribution (polydispersity index, PI). The latter is the relative variance of the size polydispersity. Cumulants does not generate a size distribution per se; it provides only a mean value and the distribution variance. With the assumption of a single-mode Gaussian function, these values can be used to construct a hypothetical size distribution.

\section{Reporting Particle Size Data}

ISO 13321:1996 gives specific recommendations for reporting of test results derived from DLS. At a minimum, the mean z-average diameter (or radius) and mean polydispersity index should be reported, along with their standard deviations based on 3 to 10 replicate measurements. The number of replicate measurements should also be reported. If a size distribution analysis algorithm is applied, then it should be identified along with any key parameter values used in the analysis. Other information that should be reported includes: particle concentration (mass or volume based, number based if known), dispersion medium composition, refractive index values 
for the particles (if other than intensity weighted distributions are reported) and the dispersion medium, viscosity value for the medium, measurement temperature, filtration or other procedure used to remove extraneous particulates/dust prior to analysis (including pore size and filter type, centrifugal force, etc.), cuvette type and size (path length), instrument make and model, scattering angle(s), and laser wavelength. Additional information that can be helpful to include in a report: measured autocorrelation $y$-intercept (amplitude), mean count rate during measurements, duration of a single measurement, and mean signal-to-noise ratio.

\section{References}

\section{General Information and Physical Property Values}

1. D.R. Lide (editor). 2006. Concentrative Properties of Aqueous Solutions: Density, Refractive Index, Freezing Point Depression, and Viscosity, in CRC Handbook of Chemistry and Physics, Internet Version 2006 (http://www.hbcpnetbase.com/), Taylor and Francis: Boca Raton, FL.

2. E.W. Lemmon, M.O. McLinden and D.G. Friend. 2005. Thermophysical Properties of Fluid Systems, in NIST Chemistry WebBook, NIST Standard Reference Database Number 69 (edited by P.J. Linstrom and W.G. Mallard). National Institute of Standards and Technology, Gaithersburg MD, 20899 (http://webbook.nist.gov/).

3. V.A. Hackley and C.F. Ferraris. 2001. The Use of Nomenclature in Dispersion Science and Technology, NIST Recommended Practice Guide, SP 960-3 (for terminology related to particle science and size measurement - available for download as a PDF file at http://www.ceramics.nist.gov/pubs/practice.htm).

4. A. Jillavenkatesa, S.J. Dapkunas and L.-H. Lum. 2001. Particle Size Characterization, NIST Recommended Practice Guide, SP 960-1 (for general information related to particle size measurement and data representation - available as a downloadable PDF file at http://www.ceramics.nist.gov/pubs/practice.htm)

5. IAPWS. 1997. Release on Refractive Index of Ordinary Water Substance as a Function of Wavelength, Temperature and Pressure, International Association for the Properties of Water and Steam (IAPWS): Erlangen, Germany. (available for download as a PDF file at http://www.iapws.org/)

6. E.D. Palik (editor). 1985. Handbook of Optical Constants of Solids, Academic Press: Orlando, FL. (see also Handbook of Optical Constants of Solids II, 1991, Academic Press: Boston)

\section{Selected References for DLS Theory, Instrumentation and Methodology}

7. A. Lomakin, D.B. Teplow and G.B. Benedek. 2005. Quasielastic light scattering for protein assembly study, Chapt. 10, pp. 153-174 in Amyloid Proteins: Methods and Protocols, Methods in Molecular Biology, vol. 299 (edited by E. M. Sigurdsson). Humana Press Inc.: Totowa, NJ.B.J.

8. B.J. Berne and R. Pecora. 2000. Dynamic Light Scattering - With Applications to Chemistry, Biology, and Physics. Dover Publications: Mineola NY. 
9. ISO 13321:1996. Particle Size Analysis - Photon Correlation Spectroscopy. International Organization for Standardization (provides information and guidance on instrumentation, sample preparation, measurement, and data analysis - available for electronic purchase and download at http://www.iso.org//).

10. ISO 22412:2008. Particle Size Analysis - Dynamic Light Scattering (DLS). Updates and extends ISO 13321:1996 (available for electronic purchase and download at http://www.iso.org/).

11. E2490-09. Standard Guide for Measurement of Particle Size Distribution of Nanomaterials in Suspension by Photon Correlation Spectroscopy (PCS). ASTM International (available for electronic purchase and download at http://www.astm.org/)

12. N.C. Santos and M.A.R.B. Castanho. 1996. Teaching Light Scattering Spectroscopy: The Dimension and Shape of Tobacco Mosaic Virus, Biophys. J. 71:1641-1646.

13. R. Finsy. 1994. Particle Sizing by Quasi-Elastic Light Scattering, Adv. Colloid Interface Sci, 52:79-143.

14. W. Brown (editor). 1993. Dynamic Light Scattering - The Method and Some Applications. Clarendon Press: Oxford.

15. R.M. Johnsen and W. Brown. 1992. An Overview of Current Methods of Analyzing QLS Data, Chapt. 6, pp. 78-91 in Laser Light Scattering in Biochemistry (edited by S.E. Harding, D.B. Sattelle and V.A. Bloomfield). The Royal Society of Chemistry: Cambridge.

16. B.B. Weiner and W.W. Tscharnuter. 1987. Uses and Abuses of Photon Correlation Spectroscopy in Particle Sizing, pp. 49-61, Chapter 3, in Particle Size Distribution: Assessment and Characterization (edited by T. Provder). American Chemical Society: Washington, DC.

17. I.D. Morrison, E.F. Grabowski and C.A. Herb. 1985. Improved Techniques for Particle Size Determination by Quasi-Elastic Light Scattering, Langmuir, 1:496-501.

\section{Glossary of Terms}

This section contains a limited glossary of terms that may be helpful to those reading and using this document.

baseline: The measured far point of a correlation function, typically determined from one or more channels of the correlator positioned at very large delay times. Essentially the square of the time-averaged scattered intensity; baseline can also be calculated from the square of the total measured photon counts over the course of the experiment divided by the total number of time intervals. For reporting purposes in this protocol the baseline is the value of $\left[g^{(2)}(\tau)-1\right]^{0.5}$ determined from the far point channels (see correlogram and signal-to-noise ratio).

correlator: A digital correlator partitions time into a series of clock intervals of small (typically sub-microsecond) duration and constructs the sums of the products of photon counts registered at 
different intervals separated by increasing delay or lag time, $\tau$. The raw product of the correlator is the intensity autocorrelation function. Alternatively, a correlator can compare the signals arriving at identical times from two different sources, a method referred to as cross-correlation.

correlation function (coefficients): The primary output or product of a digital correlator; essentially, the raw data generated by a DLS experiment that is subjected to analysis in order to derive a characteristic particle size and/or size distribution. In DLS, the correlator measures the intensity-intensity correlation, an exponentially decaying function of time with each individual data point referred to as a correlation coefficient. Also called the autocorrelation function because it compares photon counts from the same source at time $t$ with those at time $t+\tau$, where $\tau$ is the delay or lag time; it is typically baseline-normalized such that the value at very large $\tau$ approaches unity. By convention, upper case $G$ is used to represent the unnormalized function, while lower case $g$ indicates normalized values. A superscript 2 , as in $g^{(2)}(\tau)$, indicates a second order (intensity) correlation function, from which the first order (electric field) correlation function is calculated according to: $g^{(1)}(\tau) \sqrt{\beta}=\left[g^{(2)}(\tau)-1\right]^{0.5}$, where $\sqrt{\beta}$ is the $y$-intercept of the function on the right-hand side extrapolated to $\tau=0$. For non-interacting Brownian particles, $g^{(1)}(\tau)$ is related to the translational diffusion coefficient, $D$, such that $\ln g^{(1)}(\tau)=-D q^{2} \tau$ and $q=\left(4 \pi n / \lambda_{0}\right) \sin (\theta / 2)$, where $\theta$ is the scattering angle, $n$ is the refractive index of the suspending liquid at the laser wavelength and $\lambda_{0}$ is the wavelength in vacuo.

correlogram: A graphical representation of the correlation function with delay time, $\tau$, on the $x$ axis. The $y$-axis may be presented in one of several forms: (1) the normalized intensity autocorrelation, $g^{(2)}(\tau)$, for which the baseline value at $\tau=\infty$ approaches 1 and the $y$-intercept at $\tau=0$ can obtain a maximum value of 2 ; (2) $g^{(2)}(\tau)-1$, where the baseline value approaches 0 and the $y$-intercept has a maximum of 1 ; (3) $\left[g^{(2)}(\tau)-1\right]^{0.5}$, sometimes denoted as $G^{(1)}(\tau)$, which also varies from 0 to 1 ; and (4) the normalized field correlation function, $g^{(1)}(\tau)$, with the same limiting values as the previous two functions, and the only function that is instrumentindependent. Because of the exponential decay associated with correlation functions, natural logarithmic plots are occasionally used.

count rate: The average number of photons detected per unit time in kilo-counts per second (kcps).

cumulants: A method for fitting the first order autocorrelation function determined in a DLS experiment as a polynomial expansion in delay time, $\tau$, as described in ISO 13321:1996. Cumulants produces an estimate of the mean $\left(x_{P C S}\right)$ and width (polydispersity index) of the underlying, presumed Gaussian, particle size distribution.

decay rate (decay constant), $\Gamma$ : The characteristic rate at which the exponentially decaying first order autocorrelation function decreases toward its baseline. The rate of decay is related to the translational diffusion coefficient of the particles, $D$, by the relationship $\Gamma=D q^{2}$, where 
$q=\left(4 \pi n / \lambda_{0}\right) \sin (\theta / 2)$ is the modulus of the scattering vector. For a given experimental setup, $q$ is determined by the refractive index, $n$, of the suspending liquid, the laser wavelength in vacuo, $\lambda_{0}$, and the scattering angle, $\theta . \Gamma$ is in units of inverse seconds.

diffusion coefficient, D: A value that characterizes the random translational "Brownian" motion of particles in a liquid medium and which is expressed in units of distance-squared per unit time; this quantity is subsequently input to the Stokes-Einstein equation (see text) to calculate hydrodynamic size. More specifically, in DLS it is the collective diffusion of a large number of particles that is observed, and therefore interactions between particles may have to be considered; particle interactions tend to be concentration dependent.

hydrodynamic diameter, $\mathbf{d}_{\mathrm{H}}$ : The diameter of an equivalent hard sphere that diffuses in solution at the same rate as the analyte particles, as determined in DLS by application of the Stokes-Einstein equation (see text).

nanosize: For purposes of this protocol, implies that all physical dimensions are smaller than $100 \mathrm{~nm}\left(10^{-9} \mathrm{~m}\right)$.

polydispersity index (PI): Measure of the broadness of a size distribution derived from the cumulants analysis of DLS data according to ISO 13321:1996; for a single Gaussian population with standard deviation, $\sigma$, and mean size, $x_{P C S}$, then $P I=\sigma^{2} / x_{P C S}{ }^{2}$ is the relative variance of the distribution. As a rule of thumb, PI values smaller than about 0.04 are considered monodisperse.

qualification: Proof with reference material that an instrument is operating in agreement with the manufacturer's specifications.

refractive index (RI) contrast: The difference in refractive index between the particle phase and the suspending medium. In a DLS experiment, higher RI contrast translates to greater light scattering intensity, subject to other properties such as particle size, shape and concentration.

signal-to-noise ratio, $(\mathbf{S} / \mathbf{N})$ : Defined for DLS experiments in the following manner: $[$ intercept $+1 /$ basline +1$]-1$, where intercept is the value of $\left[g^{(2)}(\tau)-1\right]^{0.5}$ (see correlogram) extrapolated to $\tau=0$ and baseline is $\left[g^{(2)}(\tau)-1\right]^{0.5}$ determined from the far point channels of the correlator and should be close to 0 in magnitude.

validation: Proof with reference material that a measurement procedure is acceptable for all elements of its scope.

y-intercept: The extrapolated $\tau=0$ value for the measured correlation function. Warning: the $y$ intercept value may be reported differently, depending on the specific form of the correlation function used in its determination (see correlogram and correlation function). For reporting purposes in this protocol the $y$-intercept is determined from a plot of the function $\left[g^{(2)}(\tau)-1\right]^{0.5}$. 
z-average: Intensity-weighted average as applied to the diffusion coefficient isolated in a DLS experiment. Also, a term commonly applied to the harmonic z-average size, $x_{P C S}$, derived from a cumulants analysis of DLS data as described in ISO 13321:1996.

\title{
8. Abbreviations
}

\author{
BSA bovine serum albumin \\ DIS draft international standard \\ DLS dynamic light scattering \\ HEPA high-efficiency particulate air \\ ISO International Standards Organization \\ NIST National Institute of Standards and Technology \\ NNLS non-negative least squares (or non-negatively constrained least squares) \\ PBS phosphate buffered saline \\ PCS photon correlation spectroscopy \\ PI polydispersity index \\ QELS quasi-elastic light scattering \\ RI refractive index \\ RGD Rayleigh-Gans-Debye \\ RM reference material \\ SI Système International d'unités (French) \\ SRM Standard Reference Material ${ }^{\circledR}$
}

